\title{
The Multi-Product Price-Setting Newsvendor with Resource Capacity Constraints
}

\author{
Chase C. Murray ${ }^{\mathrm{a}, 1,}$, Abhijit Gosavi ${ }^{\mathrm{b}}$, Debabrata (Debu) Talukdar ${ }^{\mathrm{c}}$ \\ ${ }^{a}$ Department of Industrial $\mathcal{F}$ Systems Engineering, Auburn University \\ ${ }^{b}$ Department of Engineering Management $\mathcal{E}$ Systems Engineering, Missouri University of Science and Technology \\ ${ }^{c}$ Department of Marketing, University at Buffalo - The State University of New York
}

\begin{abstract}
In this paper, we consider a newsvendor problem commonly encountered in retail stores that cater to budgetsensitive shoppers. The newsvendor sells multiple products, and in addition to determining order quantities must also determine the selling price of each product sold. The demand for each product is of a stochastic nature and depends on the demands of other products, i.e., is cross-elastic. We transform the objective function into a form that is amenable to developing integer-programming solutions and then develop a numeric optimization procedure rooted in the Nelder-Mead search technique. We obtain encouraging numerical results on some small-scale and large-scale problems. To the best of our knowledge, this is the first work that produces implementable solutions for a scenario that is particularly relevant to retailers catering to budget-sensitive customers who are abundant in the current economy.
\end{abstract}

Keywords: Newsvendor, Pricing, Multiple products, Allocation, Nonlinear optimization.

\section{Introduction}

Retail markets in the USA have experienced significant increase in competitive pressure over the last decade (Zhu et al., 2009). The recent recessionary economic environment has further accentuated that pressure (Ailawadi et al., 2010; Clifford, 2010; Flatters and Willmott, 2009). As competitive pressure continues to intensify, retailers increasingly look for various strategic ways to enhance their operational efficiencies. One natural area of focus in that endeavor by retailers is how to make their inventory management practices more efficient (Ball, 2004; Levy et al., 2004; Murray et al., 2010). The fundamental challenge there for retailers is to develop a decision process that not only conforms to the realities of intrinsic resource constraints for inventory holding, but also responds to prevailing consumer demand dynamics.

So, a pertinent issue in this context becomes what characterizes contemporary consumer demand dynamics in typical retail markets. Systematic evidence suggests that such dynamics are characterized by consumers who are seeking significant variety in product choice options as well as good "values" in terms of price "deals" among those options (Gauri et al., 2008; Geyskens et al., 2010; McAlister et al., 2009; Talukdar et al., 2010; van Heerde et al., 2004). Based on price deals offered, these consumers are more inclined to shift their purchase timings and to switch among substitute product items than to exhibit any strong loyalty to any particular item. Not surprisingly, recent years have seen retailers significantly expanding their product assortments as well as offering considerable price promotions to compete for variety seeking, value conscious consumers.

\footnotetext{
${ }^{\star 2}$ Forthcoming in International Journal of Production Economics. Accepted March 7, 2012.

Email addresses: cmurray@auburn. edu (Chase C. Murray), gosavia@mst.edu (Abhijit Gosavi), dtalukda@buffalo. edu (Debabrata (Debu) Talukdar)
} 
For instance, while the average number of different items stocked by consumer packaged goods supermarket stores increased by $20 \%$ between 1970 and 1980, it has increased by more than $50 \%$ in each of the next three decades (Greenhouse, 2005; Tarnowski, 2007). Also, retailers are increasingly offering their own private label or "store brand" items as a way to satisfy both variety and value seeking by their customers (Geyskens et al., 2010). A recent survey finds that almost $85 \%$ of retail executives agree that they are paying more attention to building their store brands and $70 \%$ of them are investing more funds towards innovation of store brand products (Deloitte, 2010). Further, according to the Nielsen Company, more than 35\% of sales by the USA retailers across all product categories in 2007-2008 occurred on price promotion; that equated to more than $\$ 129$ billion in promoted dollar sales (Nielsen, 2008). Research suggests that an overwhelming proportion of such promotion sales can be attributed to consumers switching among substitute product items and shifting their purchase timings, rather than increasing their primary demand for the product categories (van Heerde et al., 2004). It is interesting and relevant to note here that, by one estimate, about $20 \%$ of all customers of consumer electronics retailer Best Buy are identified as the so called "extreme cherry pickers." These customers are "hard core value consumers" who almost exclusively purchase product items offered only on deep price discounts and exhibit very little loyalty to any particular item (Gauri et al., 2008; McAlister et al., 2009).

From a retailer's strategic perspective, the aforesaid consumer demand dynamics mean that its inventory management decision process takes into account a more volatile demand from "value conscious" consumers, which is characterized not only by the usual own-price effects but also by the cross-price effects. While the first effect measures how a consumer's demand for a product item will change in response to its own price change, the second effect measures how the demand will change in response to the changes in prices of other substitute and complementary product items (Bijmolt et al., 2005). In the context of typical multi-product, multi-category product assortments carried by retailers, this means that their inventory ordering decisions on any product item need to explicitly recognize the item's cross-price relationships with those of other related substitute and complementary product items (Duvvuri et al., 2007).

For example, a manager making inventory management decisions for desktop computers at a consumer electronics retailer like Best Buy needs to go well beyond factoring in the high penalties for stocking unsold inventories while recognizing the inherent stochastic nature of the consumer demand. The managers also need to take into account the fact that the consumer demand for a particular model of desktop computer will be influenced by its own price as well as the prices of other substitute products (e.g., other models of desktop computers and laptop computers) and complementary products (e.g., printers and monitors). Similarly, when setting the price and order quantity for a specific national brand of tortilla chips, a manager for a grocery retailer needs to consider the price and order quantity of other national and its own private label brands of tortilla chips (substitutes) as well as those of various brands of salsa (complements). And, so is the case for a manager for a clothing retailer who, when setting the price and order quantity for a dress shirt, needs to consider the price and order quantity of other dress shirts (substitutes) as well as trousers (complements).

The motivation and goal of our paper is to develop an efficient decision support model for retail managers facing realistic inventory management decision contexts like the one discussed above. Our paper extends the newsvendor modeling framework to develop such a decision support model for any retailer who sells multiple products in a given decision cycle. The newsvendor (or the newsboy) modeling framework is used extensively in modern supply chain planning of retailers and manufacturers (c.f., Wong et al. (2009); Fry et al. (2001); Cachon and Kök (2007) and references therein). Its origins can be traced to Edgeworth (1888) and numerous extensions of the newsvendor model have been studied in the literature. At the same time, while there exists some related literature on our specific research topic that we will discuss later, we believe our model is the first to develop an implementable solution that incorporates the above discussed realistic and prevailing consumer demand dynamics. Specifically, to capture the key implications of consumer demand dynamics on the retailer's decision context, our model allows the demand for each product to be stochastic, and a function of the price of the product itself as well as the prices of all other products to account for 
both own and cross price effects. The goal of the retailer's decision is to maximize its profit by selecting the optimal prices and procurement quantities for each product. The pricing and procurement decisions are made in the presence of multiple side constraints, such as budget restrictions, space limitations, inventory holding penalties, and bounds on product availability. We call this decision problem the multi-product price-setting newsvendor problem (MPPSNVP).

The remainder of this paper is organized as follows. A review of literature related to the multi-product newsvendor and the price-setting newsvendor, along with our contributions, is presented in Section 2. The mathematical formulation of our proposed model is described in Section 3. In Section 4, a general derivativefree solution approach is proposed. This approach accommodates general demand functions and general forms of uncertainty. Section 5 presents a numerical analysis to support our solution approach. Finally, conclusions and suggestions for future research are outlined in Section 6.

\section{Related Literature and Our Contributions}

In this section, we review the literature closely related to the MPPSNVP and present an analysis of our contributions. We exclude from our review research involving demand functions that are purely deterministic, those that exhibit no cross-elasticity, and problems with unconstrained pricing and assortment problems. Readers interested in those topics are referred to Yano and Gilbert (2005).

To the best of our knowledge, Shi et al. (2011) are the only authors to study the problem of jointly optimizing product prices and allocation quantities in the context of a multi-product newsvendor problem with side constraints and supplier discounts based on order quantity. They assume a particular price-sensitive demand function involving linear deterministic demand with additive uncertainty, although the cross-elasticity of price among products is not considered. Their "generalized disjunctive programming" solution approach is not guaranteed to be provide integer purchase quantities. Aydin and Porteus (2008) consider a joint pricing and quantity optimization problem in an unconstrained setting. While they do incorporate cross price effects, their analysis does not consider integer-valued allocation quantities, bounds on prices, or side constraints.

Khouja (1999) provides an elaborate review of single-period problems along with establishing 11 classifications of extensions to this problem. These extensions include the multi-product problem with side constraints and the single-product price-setting problem. The remaining literature related to the MPPSNVP may be classified into two distinct streams - the multi-product newsvendor (where prices are known) and the price-setting newsvendor (where only one product is considered). These separate streams of research are discussed below. None of these papers consider explicit product interactions.

Multi-product newsvendor: Aside from the aforementioned works of Shi et al. (2011) and Aydin and Porteus (2008), the existing studies on the multi-product newsvendor do not include product prices as decision variables. Hadley and Whitin (1963) proposed the first model for the multi-product newsvendor. They show that dynamic programming can be used to obtain integer-valued quantities for a single constraint instance (e.g., with a budget or volume constraint). However, they caution that the dynamic programming approach becomes difficult when additional constraints are incorporated. Lau and Lau (1995) and Lau and Lau (1997) study a single-period, multi-product newsvendor problem with multiple constraints. Moon and Silver (2000) propose a dynamic programming approach for solving the single-period multi-product newsvendor problem with a budgetary side constraint. They note that this approach can become computationally intensive if the number of products or the budget are large, and propose two heuristic approaches to address large-scale problems.

Abdel-Malek et al. (2004), Abdel-Malek and Montanari (2005a), and Abdel-Malek and Montanari (2005b) consider a multi-product newsvendor whose objective is to minimize expected costs related to procurement and salvage/disposal, while maximizing revenue. A budget constraint is incorporated via Lagrangian relaxation. These papers consider demand uncertainty to be either uniformly or exponentially 
distributed, or each product may have a unique "general" distribution. Closed-form solutions are provided for the first two cases, while a heuristic approach is provided for the general case. Abdel-Malek et al. (2008) address the so-called "gardener problem," where agricultural crops are planted on an acreage, and each crop may produce a random yield (based on weather conditions, for example). Their earlier solution approaches are refined to solve this problem. Abdel-Malek and Areeratchakul (2007) convert the objective function into a quadratic function and consider three types of side constraints: budget, resource capacity, and lower bounds on order quantity. They consider uniform, exponential, normal, and general demand distributions.

Niederhoff (2007) proposes a separable programming formulation for the "newstand" problem (the multi-product newsvendor problem with multiple constraints). A "binary solution method" for the multiproduct newsvendor problem is proposed by Zhang et al. (2009), which is applicable to general forms of demand functions. Taleizadeh et al. (2009) assume that product demand follows a Poisson distribution and consider order quantities to be integer-valued. They model this as a bi-objective problem, where the first objective is to maximize profits and the second objective is to maximize service level (which is the ratio of expected satisfied demand to expected demand). A goal programming approach, followed by a genetic algorithm, is used to solve the problem in which a constraint on warehouse space is included. Özler et al. (2009) address the single-period multi-product newsvendor problem with a value at risk (VaR) constraint to incorporate the risk of the newsvendor earning less than a pre-specified target profit. Zhang (2010) studies a multi-product newsvendor problem where discounts are provided based on the quantity ordered. Lagrangian relaxation is used to solve this problem in which a budget constraint is also incorporated.

Of the aforementioned multi-product newsvendor models, only Moon and Silver (2000) and Taleizadeh et al. (2009) propose approaches that guarantee integer-valued order quantities.

Price-setting newsvendor: Also related to the MPPSNVP is the single-product price-setting newsvendor problem, first studied by Whitin (1955). This single-product problem has also been addressed by Petruzzi and Dada (1999), Agrawal and Seshadri (2000), Monahan et al. (2004), Bisi and Dada (2007), and Chen and Bell (2009).

More recent treatments of this problem focus on risk aversion. For example, Chen et al. (2009) and Xu (2010) consider the problem with conditional value at risk (CVaR) constraints. Yang et al. (2010) consider two objectives, namely maximizing the probability of exceeding a profit threshold and maximizing the probability of exceeding a revenue threshold. Shi et al. (2010) consider a firm with a profit target.

Our contributions:. Our work contributes to the literature in the following ways:

- To the best of our knowledge, ours is among the first newsvendor problem studies that incorporates cross-price elasticity of demand with multiple products where both pricing and ordering quantities are decision variables. Additionally, this problem is solved in the presence of multiple side constraints.

- From a practical perspective, it is imperative for the retailer to have integer-valued ordering quantities. Hadley and Whitin (1963) mention that, for large quantities, rounding of fractional solutions to integer solutions may be acceptable. However, they also state that for relatively low quantities, rounding may lead to results that are far from optimal. In fact, rounding in this setting may even lead to infeasible solutions. We propose a solution approach to the MPPSNVP that guarantees integer-valued product quantities.

- We propose a novel method for transforming the profit function into a mathematical form that is amenable to solution via standard mixed integer programming techniques. From the perspective of operations research, this is an attractive feature since it opens the avenue for solutions using numerous other techniques. In addition, our approach is also applicable to a multi-product newsvendor problem in which prices are fixed. 


\section{Model Development}

In this section, we first discuss our model's underlying decision context and then present its analytical formulation, along with its transformation amenable to our solution approach.

\subsection{Decision Context}

Our model is formulated from the perspective of category management decisions by retailers. Specifically, how a retailer, who sells multiple product items in a given focal category, should decide about the optimal procurement quantities and prices of the items in any single decision cycle, contingent on the realities of consumer demand dynamics as well as internal resource constraints. As is typical in the existing literature on optimizing product procurement quantities (e.g., Aydin and Porteus (2008); Murray et al. (2010); Martínez-de-Albéniz and Roels (2011)), we assume that the composition of the product assortment offered by the retailer in the focal category has already been selected based on the issues of risk-pooling across substitute and complementary product items. Specifically, we consider the retailer selling a pre-selected assortment of $n(n>1)$ distinct product items in the focal product category.

To capture the key elements of consumer demand dynamics in the retailer's decision context, we assume that the demand for each of the $n$ product items in the focal category to be stochastic on any time period, and a function of its own price as well as the prices of all other product items in the focal category to account for both own and cross price effects. In other words, the realities of consumer demand dynamics imply that these $n$ product items in the focal category are inter-related in their demand as substitutes and/or complements from a consumption perspective. In the context of a consumer electronic retailer like Best Buy, an example of such a focal category of product items would be the various models of computers. Here, we can expect to find only a substitute relationship among the product items in the category. On the other hand, the focal category could consist of not only the various models of computers, but also of various computer accessories (e.g., printers). In that case, we can expect to find both substitute and complementary relationships among the product items in the category.

The practical realities of the retailer's decision context also require that the retailer's pricing and procurement decisions are made in the presence of multiple marketplace and resource constraints (Yang and Chen, 1999; Murray et al., 2010). Specifically, competitive market forces will mean that the retailer will face a vector of allowable selling prices for each product item. Additionally, the retailer may encounter upper and lower limits on the procurement quantities of product items. For example, upper bounds may exist for items in limited supply, while contractual obligations with vendors may impose lower bounds on the minimum required procurement quantities for other items. The retailer may also face a variety of resource constraints. Examples include budget or financial restrictions that dictate the maximum amount spend on product procurement, or physical facility size limitations that permit a maximum volume of goods that may be displayed or stored. The number and nature of these resource constraints may vary by retailer (e.g., a clothing retailer would not be constrained by a volume of refrigerated storage space).

The retailer's objective is to maximize expected net profit - based on the revenue generated from the sale of the products in the focal category, the costs of procuring the items, and holding costs of unsold items - subject to the aforementioned constraints.

\subsection{Analytical Formulation}

We now present the mathematical representation of the retailer's optimization decision. The following notation is employed:

- $n$ : Number of product items in the focal category.

- $c_{i}$ : Procurement cost for each unit of product $i$. 
- $h_{i}$ : Holding cost for each unsold unit of product $i$. If $h_{i}<0$, this represents a salvage value.

- $p_{i}$ : Selling price for each unit of product $i$. This is a decision variable.

- $\mathbf{p}$ : Vector of selling prices for all product items, such that $\mathbf{p}=\left\{p_{1}, p_{2}, \ldots, p_{n}\right\}$.

- $P_{i}$ : Discrete set of allowable selling prices for the product items, such that $p_{i} \in P_{i}$.

- $q_{i}$ : Integer number of units of product $i$ to stock/procure. This is a decision variable.

- $\mathbf{q}$ : Vector of stocked quantities for all product items, such that $\mathbf{q}=\left\{q_{1}, q_{2}, \ldots, q_{n}\right\}$.

- $L_{i}\left(U_{i}\right)$ : Lower (upper) bound on $q_{i}$.

- $R_{j}$ : Available amount of some resource $j \in J$, where $J$ is a set of all constrained resources.

- $r_{i j}$ : Amount of resource $j$ consumed by product $i$.

The demand for a product $i$, denoted as $D_{i}(\mathbf{p})$, is a function of prices of all product items, such that ownand cross-price effects are captured. This function contains both deterministic and stochastic components. Rather than requiring a particular functional form for $D_{i}(\mathbf{p})$, our proposed mathematical model accommodates a wide variety of own- and cross-price sensitive demand functions. The retailer's choice of the most appropriate demand function may be motivated by, for example, the manner in which complementary and/or substitution effects are best reflected for the realities of the retailer's particular situation. In Section 5 we provide a numerical analysis for some particular forms of $D_{i}(\mathbf{p})$ for demonstration purposes.

The retailer's objective function captures the profit generated from the sale of stocked units, as well as a penalty for unsold inventory. The profit function may be written as:

$$
\begin{aligned}
\pi(\mathbf{p}, \mathbf{q}) & =\sum_{i=1}^{n}\left[\left(p_{i}-c_{i}\right) q_{i}-\left(p_{i}+h_{i}\right) \sum_{d=0}^{q_{i}}\left(q_{i}-d\right) \operatorname{Pr}\left\{D_{i}(\mathbf{p})=d\right\}\right] \\
& =\sum_{i=1}^{n}\left[\left(p_{i}-c_{i}\right) q_{i}-\left(p_{i}+h_{i}\right) \sum_{d=0}^{q_{i}} \operatorname{Pr}\left\{D_{i}(\mathbf{p}) \leq d\right\}\right] \\
& =\sum_{i=1}^{n}\left[\left(p_{i}-c_{i}\right) q_{i}-\left(p_{i}+h_{i}\right) \sum_{d=0}^{q_{i}} F_{i}(\mathbf{p}, d)\right]
\end{aligned}
$$

where $\mathbf{p}$ and $\mathbf{q}$ are vectors of decision variables, and $F_{i}(\mathbf{p}, d)$ represents the cumulative distribution function (CDF) of demand for product $i$.

The optimization model for the MPPSNVP may be stated as:

$$
\begin{aligned}
\operatorname{Max} & \pi(\mathbf{p}, \mathbf{q}) \\
\text { s.t. } & L_{i} \leq q_{i} \leq U_{i} \quad \forall i=1,2, \ldots, n, \\
& \sum_{i=1}^{n} r_{i j} q_{i} \leq R_{j} \quad \forall j \in J, \\
& p_{i} \in P_{i} \quad \forall i=1,2, \ldots, n, \\
& q_{i} \geq 0 \text { and integer } \quad \forall i=1,2, \ldots, n .
\end{aligned}
$$

Constraint (2) captures the lower and upper bounds on the quantity of each product that may be purchased. These bounds may reflect contractual obligations with suppliers that require the newsvendor to purchase 
a minimum quantity, or limitations on product availability. Constraints (3) represent limitations on the newsvendor's resources. For example, these constraints may reflect budget restrictions or space limitations. Constraint (4) indicates that the prices for each product must be chosen from a discrete set, while constraint (5) restricts the order quantity of each product to be integer-valued.

Because this paper focuses on the problem of product allocation and pricing, rather than on product selection, the lower bound of allocation quantity for each product is assumed to be at least $1\left(L_{i} \geq 1\right)$. In the absence of an explicit (exogenously-determined) upper bound, it is straightforward to calculate an effective upper bound for product $i$. One approach for determining such an upper bound would be to calculate the maximum possible demand for product $i$. Another option would be to use the $R_{j}$ constraints to determine the maximum quantity that could be purchased (in the case of a budget constraint) or could be stored (in the case of volume restrictions).

\subsubsection{Transformed Profit Function}

The aforementioned model presents an optimization challenge in that the objective (profit) function contains the decision variable $q_{i}$ in the range of summation. As such, in its present form, it is not amenable to traditional integer programming solution procedures. To address this issue, we propose a transformation of the profit function, $\pi(\mathbf{p}, \mathbf{q})$, by introducing the indicator variable, $z_{i d}$, defined as:

$$
z_{i d}= \begin{cases}1 & \text { if } d \leq q_{i} \\ 0 & \text { if } d>q_{i}\end{cases}
$$

for all $i \in\{1, \ldots, n\}$ and $d \in\left\{0,1, \ldots, U_{i}\right\}$. In other words, $z_{i d}=1$ for all $d$ such that $d$ does not exceed the allocation quantity of product $i$, which is itself a decision variable. In the case that $U_{i}$ is not exogenouslydetermined, calculation of tight effective upper bounds will serve to reduce the number of decision variables in the model.

With the incorporation of $z_{i d}$, the profit function may be rewritten as follows:

$$
\pi(\mathbf{p}, \mathbf{q})=\sum_{i=1}^{n}\left[\left(p_{i}-c_{i}\right) q_{i}-\left(p_{i}+h_{i}\right) \sum_{d=0}^{L_{i}} F_{i}(\mathbf{p}, d)-\left(p_{i}+h_{i}\right) \sum_{d=L_{i}+1}^{U_{i}} F_{i}(\mathbf{p}, d) z_{i d}\right] .
$$

To justify this transformation, we first note that $q_{i} \geq L_{i}$, by assumption. Therefore, holding costs will necessarily be incurred if demand for product $i$ does not exceed $L_{i}$. Next, because $q_{i} \leq U_{i}$ (also by assumption), we need only consider demand values up to $U_{i}$. For demand between $L_{i}+1$ and $U_{i}$, a holding cost is only incurred if the allocation quantity exceeds demand (i.e., if $z_{i d}=1$ ).

To establish appropriate values for $z_{i d}$, the following constraints must be included:

$$
\begin{gathered}
\sum_{d=L_{i}+1}^{U_{i}} z_{i d}=q_{i}-L_{i} \quad \forall i=1,2, \ldots, n, \\
z_{i, d-1} \geq z_{i d} \quad \forall i \in\{1, \ldots, n\}, d \in\left\{L_{i}+2, \ldots, U_{i}\right\}, \\
0 \leq z_{i d} \leq 1 \quad \forall i \in\{1, \ldots, n\}, d \in\left\{L_{i}+1, \ldots, U_{i}\right\} .
\end{gathered}
$$

These constraints ensure that $z_{i d}=1$ if demand $d$ does not exceed the allocation quantity $q_{i}$. Otherwise, if demand exceeds supply, no holding cost should be incurred (i.e., $z_{i d}=0$ ). Constraint (7) may be best described via an example. Suppose $L_{i}=3$ and $U_{i}=12$. Furthermore, suppose that $q_{i}$ takes a value of 7 in a feasible solution. The first $L_{i}=3$ allocated/purchased units are automatically subject to holding costs by virtue of the $\sum_{d=0}^{L_{i}} F_{i}(\mathbf{p}, d)$ term in equation (6). For allocation quantities between $L_{i}+1$ and $U_{i}$, exactly 
$q_{i}-L_{i}=7-3=4$ units are subject to holding costs. Thus, $z_{i 4}+\ldots+z_{i 12}=4$. Constraint (8) will ensure that $z_{i 4}=z_{i 5}=z_{i 6}=z_{i 7}=1$. Constraint (8) captures the restriction that if $z_{i d}=1$ then $z_{i, d-1}$ must also equal 1 . Note that, since $F_{i}(\mathbf{p}, d)$ is a non-decreasing function in $d$, this constraint is not explicitly required (the objective function will attempt to avoid assigning $z_{i d}>0$ for larger values of $d$, since these values are associated with larger values of $F_{i}(\mathbf{p}, d)$ ). However, the relationships expressed in this constraint should serve to improve the efficiency of the solution approach. Constraint (9) restricts $z_{i d}$ to the appropriate range of values.

One pleasant property of this formulation is that the explicit restriction of $z_{i d}$ to be binary is not required, as the above constraints will necessarily result in binary values for these indicator variables. Thus, although we are adding decision variables to the model, they are continuous. Although we did not perform a fullscale numerical analysis specifically to quantify the performance impact of using binary versus continuous indicator variables, our initial testing (to validate the model) revealed that problems may be solved roughly twice as fast if $z_{i d}$ is defined as a continuous variable.

Our mathematical model may now be expressed as maximizing (6) subject to constraints (2) - (5) and (7) - (9). We shall refer to this problem as MIP-1, and restate it as:

$$
\begin{array}{ll}
\text { (MIP-1): } \quad \text { Max } \quad & \pi(\mathbf{p}, \mathbf{q})=\sum_{i=1}^{n}\left[\left(p_{i}-c_{i}\right) q_{i}-\left(p_{i}+h_{i}\right) \sum_{d=0}^{L_{i}} F_{i}(\mathbf{p}, d)-\left(p_{i}+h_{i}\right) \sum_{d=L_{i}+1}^{U_{i}} F_{i}(\mathbf{p}, d) z_{i d}\right] \\
\text { s.t. } \quad & L_{i} \leq q_{i} \leq U_{i} \quad \forall i=1,2, \ldots, n, \\
& \sum_{i=1}^{n} r_{i j} q_{i} \leq R_{j} \quad \forall j \in J, \\
& \sum_{d=L_{i}+1}^{U_{i}} z_{i d}=q_{i}-L_{i} \quad \forall i=1,2, \ldots, n, \\
& z_{i, d-1} \geq z_{i d} \quad \forall i \in\{1, \ldots, n\}, d \in\left\{L_{i}+2, \ldots, U_{i}\right\}, \\
& p_{i} \in P_{i} \quad \forall i=1,2, \ldots, n, \\
& q_{i} \geq 0 \text { and integer } \quad \forall i=1,2, \ldots, n, \\
& 0 \leq z_{i d} \leq 1 \quad \forall i \in\{1, \ldots, n\}, d \in\left\{L_{i}+1, \ldots, U_{i}\right\} .
\end{array}
$$

\section{Solution Approach}

Before beginning our discussion on the proposed solution approach, it may be insightful to explore the structure of the MPPSNVP. First, due to a combination of the effects of cross-price elasticities of demand, integer order quantities, and resource capacity constraints (e.g., budget and space limitations), the objective function value is both nonlinear and non-convex. For example, Figure 1 shows the objective function value for each admissible combination of selling prices for a two-product problem. Due to the imposition of integer order quantity restrictions and side constraints, a closed-form solution to problem MIP-1 is not available.

Fortunately, there are some structural properties of problem MIP-1 that may be exploited. First, the constraints are linear. Second, suppose we temporarily limit our focus to one particular realization of selling prices. Given a selling price for each product, these prices (temporarily) become parameters rather than decision variables. As a result, the only decision variables in the objective function are $q_{i}$ (allocation quantity) and $z_{i d}$ (an auxiliary decision variable). Due to the assumption that demand is a function of all product prices, the CDF of demand will not be a function of the $q_{i}$ or $z_{i d}$ decision variables. Therefore, given $a$ particular selling price for each product, the objective function $\pi(\mathbf{p}, \mathbf{q})$ is linear, as no decision variables are 


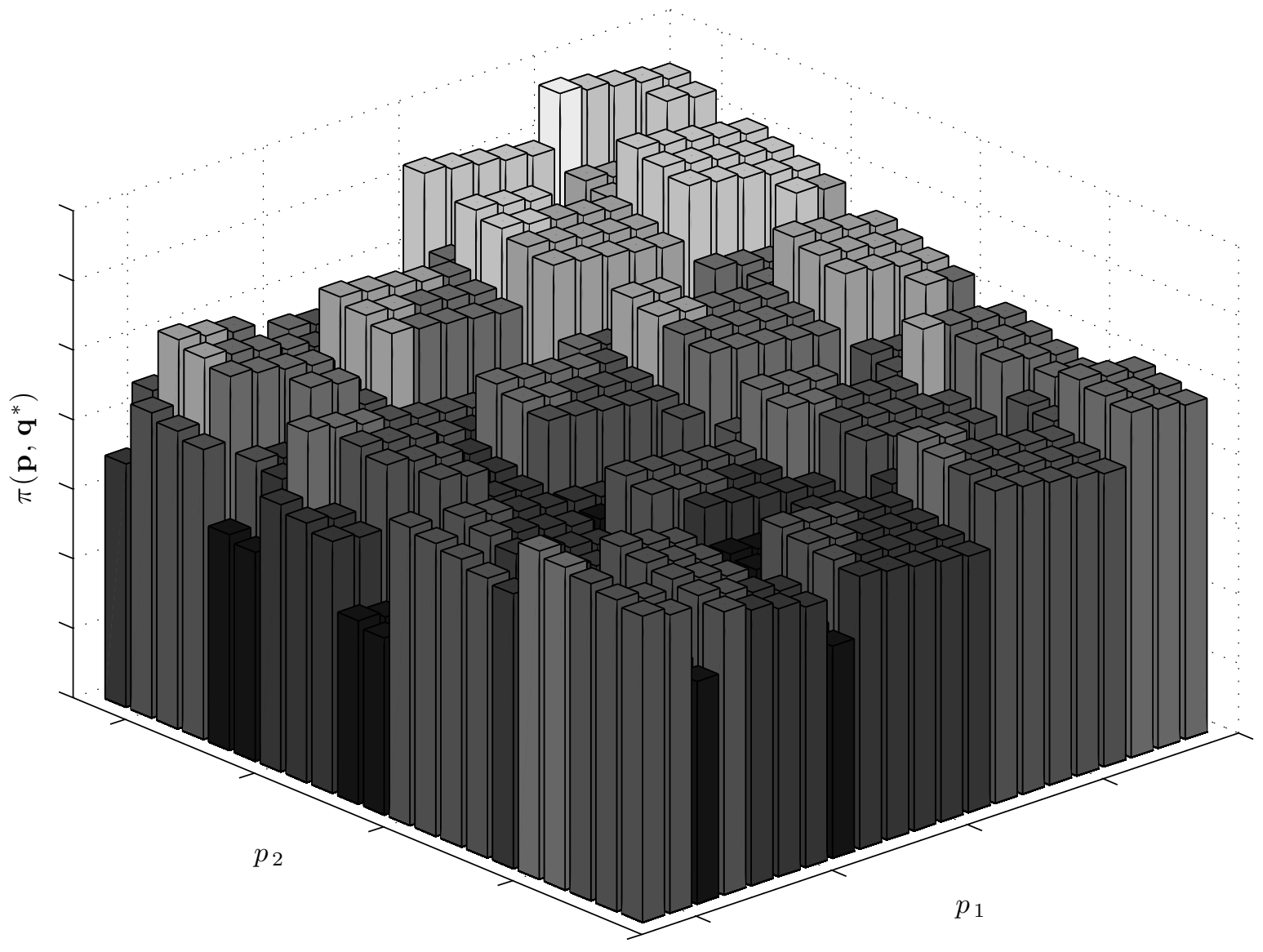

Figure 1: A plot of the optimal objective function value for each distinct price combination of a two-product problem.

multiplied. In other words, for a fixed vector of product prices, $\mathbf{p}$, the MPPSNV problem becomes separable in product quantity. A similar observation regarding this separability was identified by Niederhoff (2007) in the case of a multiproduct non-price-setting newsvendor.

Although we are interested in determining optimal product prices and quantities, this observation is still relevant to our solution approach. Thus, even though our profit function incorporates cross-price elasticities, the profit function is linear in the $q_{i}$ decision variables for a given (fixed) vector of prices. Using this observation, we define problem MIP-2 to be a modified version of problem MIP-1, where the values of $p_{i}$ are (temporarily) defined as parameters rather than decision variables.

The benefit of model MIP-2 is that it may be solved as a linear integer program to determine the optimal allocation quantities for a given vector of selling prices. Since the MPPSNV problem requires the optimization of both the allocation quantities and the selling prices, problem MIP-2 alone is insufficient. Therefore, our approach iteratively investigates candidate price vectors, solving problem MIP-2 to determine optimal procurement quantities for each given price vector. In each iteration, these candidate prices are chosen via a derivative-free method, as described below. 


\subsection{A Multidirectional Search Heuristic for the MPPSNVP}

The proposed derivative-free approach for the MPPSNVP is inspired by the multidirectional search algorithm of Torczon (1991), who developed her approach for solving unconstrained continuous optimization problems. In contrast to the well-known Nelder-Mead simplex algorithm (Nelder and Mead, 1965), which searches in only a single direction in each iteration, Torczon's method searches across all $n$ directions simultaneously. The general idea of the solution approach proposed in this paper is as follows. In each iteration of the procedure, $n+1$ candidate vectors of prices are considered, forming a simplex, thus allowing a simultaneous search over price changes for all $n$ products. For each candidate price vector, problem MIP-2 is solved. This yields the optimal procurement quantities at this particular price, $\mathbf{q}^{*}$, and the corresponding optimal profit function value at the given prices.

To facilitate computer programming of the proposed heuristic, we define the $n+1$ vectors of prices in terms of both their indices and their values. The set of allowable prices for product $i$ is given by the set $P_{i}$, such that there are $\left|P_{i}\right|$ possible prices for product $i$. The vectors of price indices are denoted $\mathbf{V}^{0}, \mathbf{V}^{1}, \ldots, \mathbf{V}^{n}$, where $\mathbf{V}^{0}$ is called the anchor vector. Thus, for any vector $0 \leq j \leq n, \mathbf{V}^{j}=\left[v_{1}^{j}, v_{2}^{j}, \ldots, v_{n}^{j}\right]$, where $v_{i}^{j}$ is the price index for product $i$ and such that $v_{i}^{j} \in\left\{1,2, \ldots,\left|P_{i}\right|\right\}$. Each vector of price indices may be mapped to the corresponding price values, as follows. Consider price index vector $\mathbf{V}^{j}$. The corresponding price value for a particular product $i$ is given by $\mathbf{P}_{i}\left(v_{i}^{j}\right)$. Thus, the vector of price values for all products, according to the indices given by $\mathbf{V}^{j}$, is obtained by $\mathbf{P}=\left[\mathbf{P}_{1}\left(v_{1}^{j}\right), \mathbf{P}_{2}\left(v_{2}^{j}\right), \ldots, \mathbf{P}_{n}\left(v_{n}^{j}\right)\right]$. The five-step heuristic for solving the MPPSNVP is described in the remainder of this section.

Step 1: Randomly generate an anchor vector of price indices.

In this step we establish the initial anchor vector, $\mathbf{V}^{0}$, within the interior of the solution space for price indices. As such, $\mathbf{V}^{0}=\left[v_{1}^{0}, v_{2}^{0}, \ldots, v_{n}^{0}\right]$, where $v_{i}^{0}$ is the price index for product $i$. The corresponding price for product $i$ is given by $\mathbf{P}_{i}\left(v_{i}^{0}\right)$. Thus, $\mathbf{P}=\left[\mathbf{P}_{1}\left(v_{1}^{0}\right), \mathbf{P}_{2}\left(v_{2}^{0}\right), \ldots, \mathbf{P}_{n}\left(v_{n}^{0}\right)\right]$ represents the vector of product prices associated with the initial anchor vector of product price indices. Problem MIP-2 is solved to find $\mathbf{q}^{*}$, the optimal procurement quantity for the prices given by $\mathbf{P}$. The corresponding objective function value, $\pi\left(\mathbf{P}, \mathbf{q}^{*}\right)$ is saved as our best-known profit value, denoted by $\pi^{\prime}$. The pseudocode for this step is as follows:

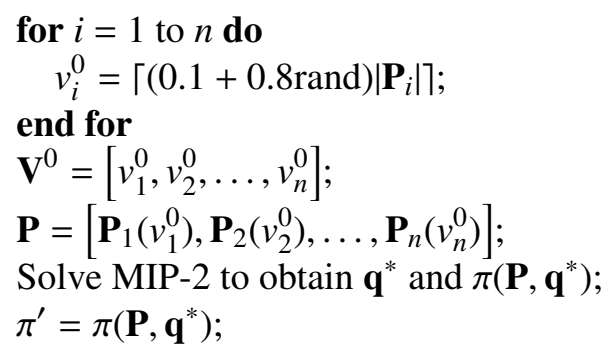

Step 2: Generate the remaining $n$ price indices to form the initial simplex.

We must now create $n$ unique price index vectors to complete the initial simplex. Each of these vectors will differ from the anchor vector in only one price, and this price will be associated with an index that is \pm 1 element from the corresponding element of the anchor. To assist in this effort, we make use of the $n$-element unit vector $\mathbf{e}_{j}$, which contains $n-1$ zeros and a 1 in position $j$. Pseudo-code for this step is as follows:

$$
\text { for } \begin{aligned}
j & =1 \text { to } n \mathbf{d o} \\
\mathbf{V}^{j} & = \begin{cases}\mathbf{V}^{0}+\mathbf{e}_{j} & \text { with probability } 0.5 \\
\mathbf{V}^{0}-\mathbf{e}_{j} & \text { with probability } 0.5\end{cases}
\end{aligned}
$$

end for 
Step 3: Evaluate the current simplex and test for possible expansion.

In Step 3 we solve problem MIP-2 to determine if the $n$ non-anchor vertices of the current simplex produce an objective function value that exceeds that of the anchor vertex. This step also involves an expansion procedure that generates additional candidate vertices. These vertices are established by moving along the direction from the anchor to each of the other initial vertices of the current simplex. The magnitude of the movement in each direction is dictated by a set of increasing scaling parameters, denoted as $\Gamma^{\exp }$. Through preliminary testing, we have found that the following elements of $\Gamma^{\exp }$ work well, although other values may be more appropriate for certain problems:

$$
\Gamma^{\exp }=\left[0, \max \left\{\left\lfloor 0.15 \min _{i}\left\{\left|\mathbf{P}_{i}\right|\right\}\right\rfloor, 1\right\}, \max \left\{\left\lfloor 0.30 \min _{i}\left\{\left|\mathbf{P}_{i}\right|\right\}\right\rfloor, 2\right\}\right] .
$$

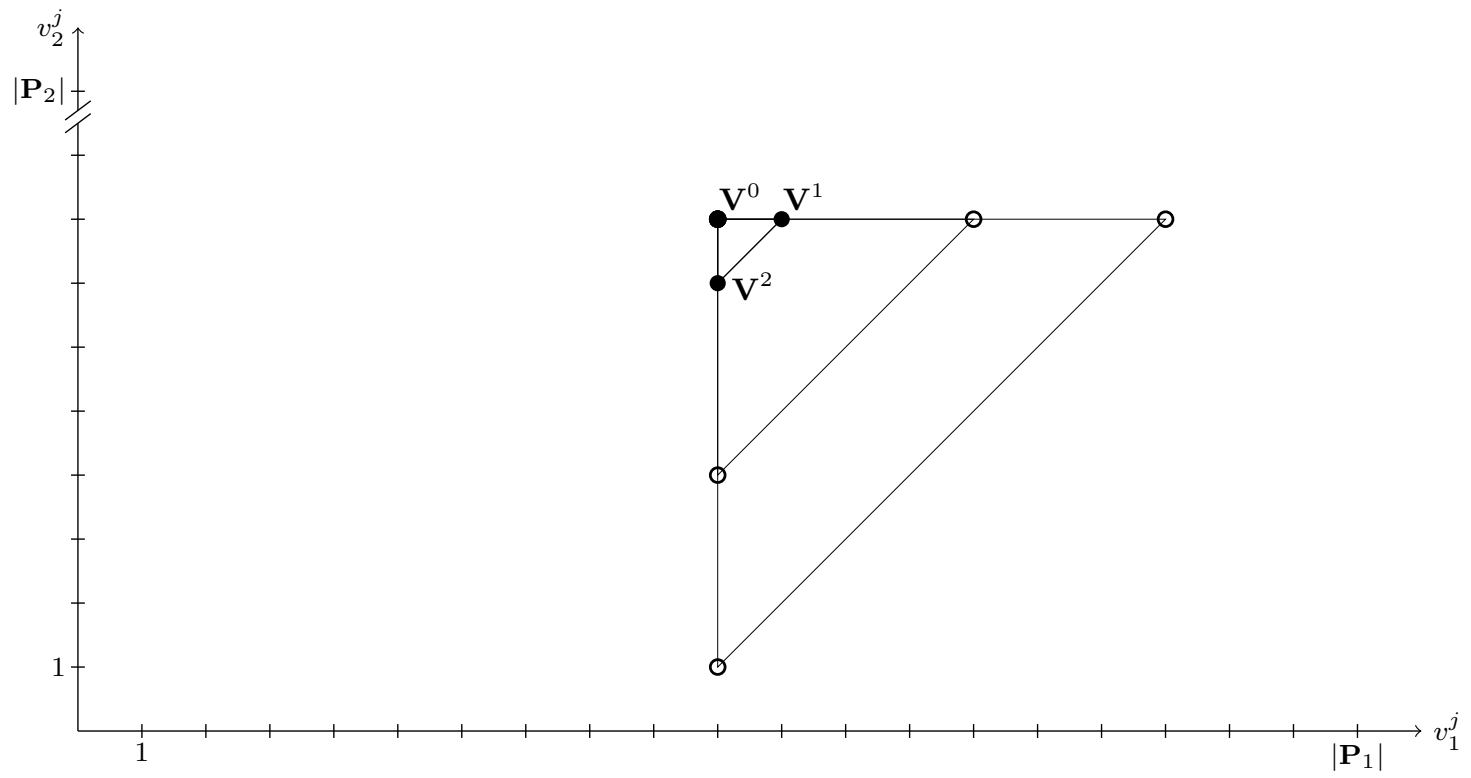

Figure 2: Representative expansion of the simplex defined by vectors $\mathbf{V}^{0}, \mathbf{V}^{1}$, and $\mathbf{V}^{2}$.

Figure 2 demonstrates an initial simplex and two expanded simplices for a two-product problem. Pseudo-code for Step 3 is as follows:

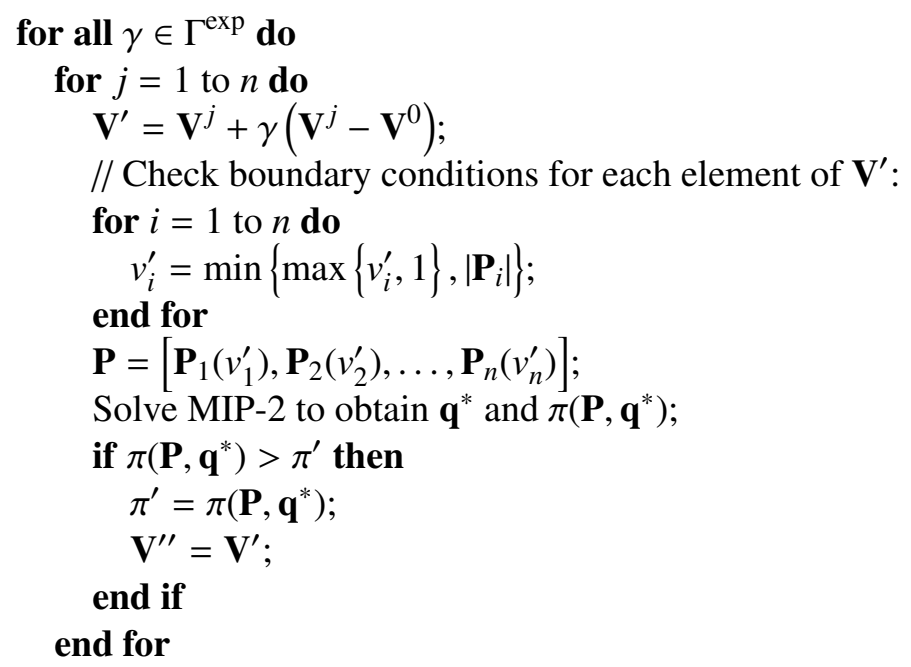




\section{end for}

If an improved objective function value is obtained in Step 3, the procedure skips to Step 5. Otherwise, continue to Step 4.

Step 4: Reflect the current simplex and test for expansion in a new direction.

If no improvement to the objective function values was found in Step 3, we will reflect the current simplex about the anchor vector, $\mathbf{V}^{0}$, and investigate candidate solutions in the opposite direction, as demonstrated in Figure 3. The updated $n$ non-anchor vertices of the reflected simplex are given by:

$$
\mathbf{V}^{j}=\mathbf{V}^{0}-\left(\hat{\mathbf{V}}^{j}-\mathbf{V}^{0}\right) \quad \forall j=1, \ldots, n,
$$

where $\hat{\mathbf{V}}^{j}$ represents vertex $j$ of the current simplex.

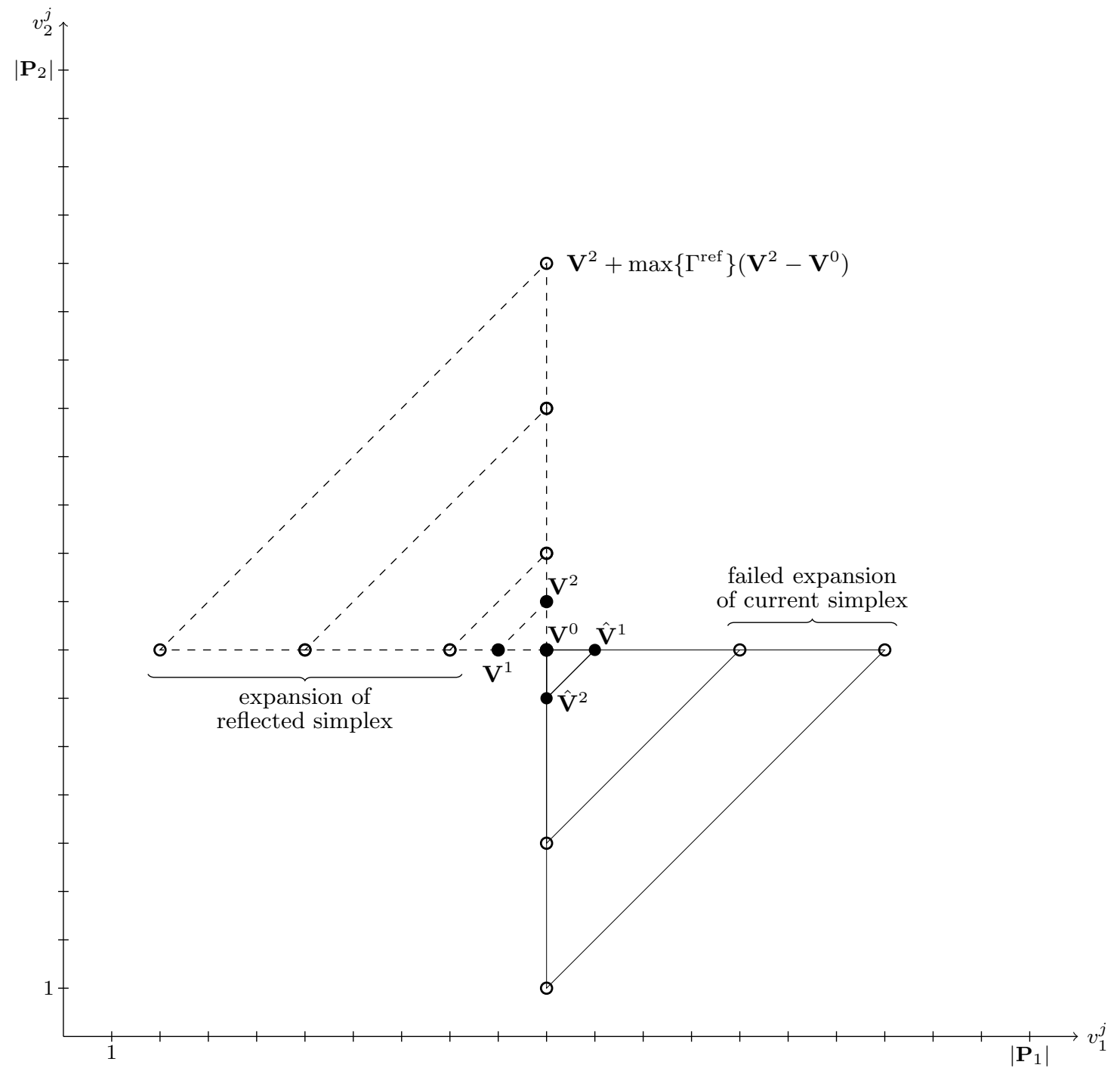

Figure 3: Representative reflection of the simplex defined by vectors $\mathbf{V}^{0}, \hat{\mathbf{V}}^{1}$, and $\hat{\mathbf{V}}^{2}$. This reflected simplex may then be expanded with a magnitude according the values of $\Gamma^{\mathrm{ref}}$.

The objective function value associated with the new (reflected) simplex is calculated, and expansion of this reflected simplex is also tested. This procedure is exactly as in Step 3, with the exception that 
$\Gamma^{\mathrm{exp}}$ should be replaced by $\Gamma^{\mathrm{ref}}$, which is defined as follows:

$$
\begin{aligned}
\Gamma^{\mathrm{ref}}= & {\left.\left[0, \max \left\{\mid 0.05 \min _{i}\left\{\left|\mathbf{P}_{i}\right|\right\}\right], 1\right\}, \max \left\{\mid 0.20 \min _{i}\left\{\left|\mathbf{P}_{i}\right|\right\}\right\rfloor, 2\right\}, } \\
& \left.\max \left\{\left\lfloor 0.35 \min _{i}\left\{\left|\mathbf{P}_{i}\right|\right\}\right\rfloor, 3\right\}\right] .
\end{aligned}
$$

As with $\Gamma^{\mathrm{exp}}$, the number and value of these elements may be modified.

Step 5: Terminate or define new simplex.

The procedure may be terminated if either of the following conditions is met:

1. Neither Steps 3 nor 4 yielded an objective function value that was better than that found at the current anchor vector. In this case, the procedure has encountered a local optimal from which it cannot immediately escape. The procedure may be repeated, starting with Step 1, where a new initial solution is randomly generated. This provides the heuristic the opportunity to escape the local optimal and to explore more of the solution space.

2. A pre-specified runtime limit has been reached. Such a time-based (or, equivalently, an iterationbased) condition is commonly applied in simulated annealing, tabu search, genetic algorithm, and ant colony optimization heuristics. Recent applications of these heuristics to a variety of optimization problems are given by Corman et al. (2010), Leung et al. (2010), Moin et al. (2011), and Zhang et al. (2010). This termination condition is employed by these popular heuristics because the problems to which they are applied do not offer bounds that would form a basis for an optimality gap. Likewise, there is no bound for the MPPSNVP.

These termination criteria work in tandem, allowing the procedure to escape locally-optimal solutions while also recognizing that decision makers cannot wait indefinitely to implement a solution. When the termination criteria are met, the anchor corresponding to the best-known value of $\pi^{\prime}$ should be reported as the best-known price vector.

Otherwise, if a termination criterion has not been met, use the simplex vertex associated with the best objective function value as the anchor for a new simplex. We will denote this vertex as $\mathbf{V}^{\prime \prime}$.

If $\mathbf{V}^{\prime \prime}$ is associated with the maximum expansion of the previous simplex (i.e., when $\gamma=\max \left\{\Gamma^{\exp }\right\}$ or $\gamma=\max \left\{\Gamma^{\mathrm{ref}}\right\}$ ), the previous simplex is effectively moved to the location of the new anchor vertex, as shown in Figure 4. This action is motivated by the observation that the objective function value appears likely to continue improving in the current direction of expansion. This new simplex may be found as follows:

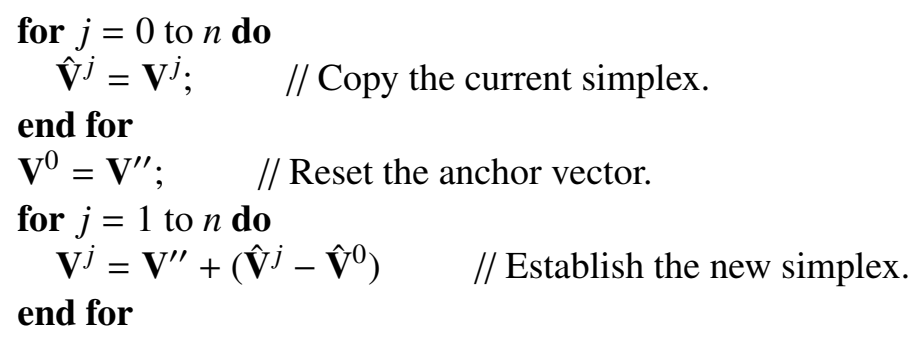

However, if $\mathbf{V}^{\prime \prime}$ is not associated with the maximum expansion, a randomly-generated simplex is created from the new anchor point, as shown in Figure 5. In this case, the objective function is not showing evidence of improvement at the extreme expansion of the simplex, and therefore proceeding in a new direction may be fruitful.

$\mathbf{V}^{0}=\mathbf{V}^{\prime \prime} ; \quad / /$ Reset the anchor vector. 


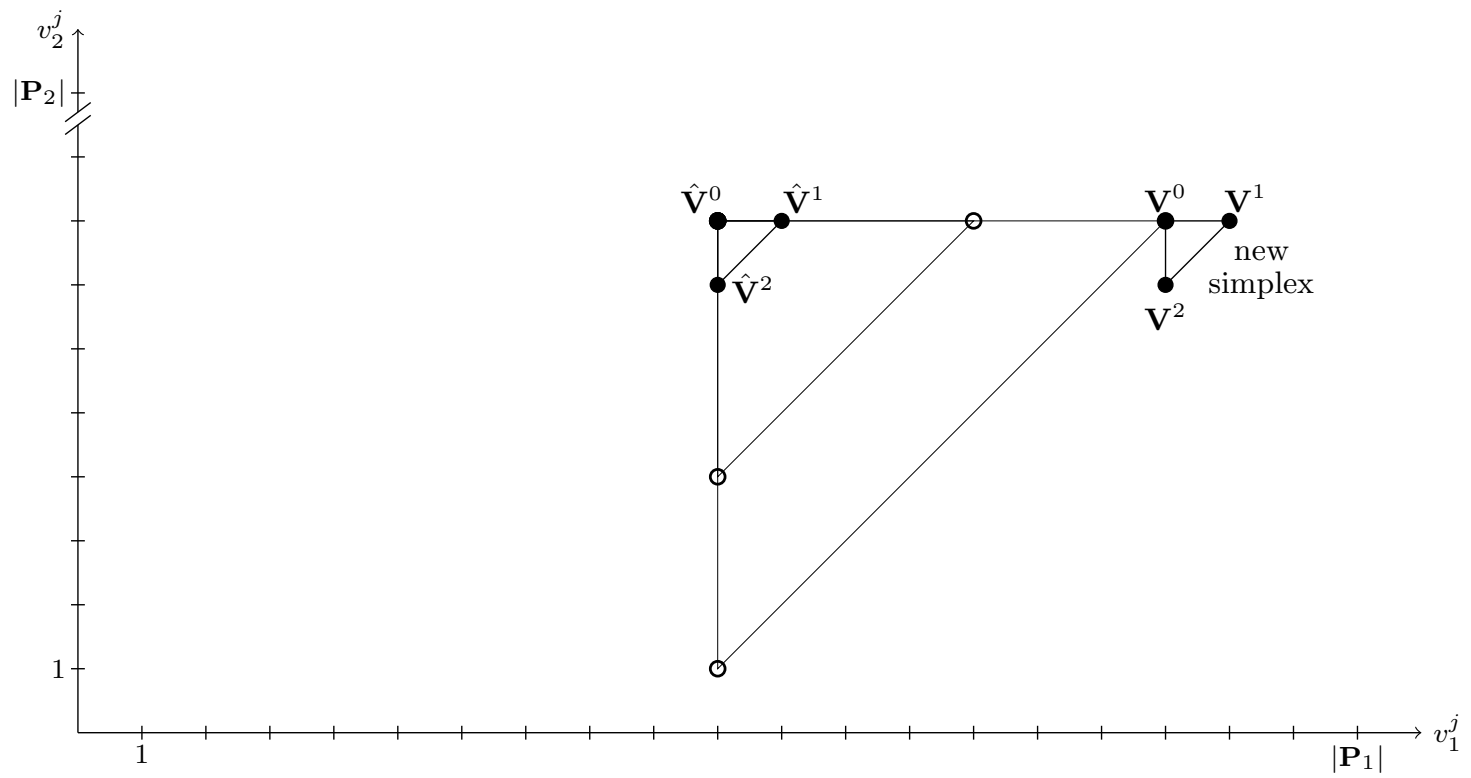

Figure 4: Vertex $\mathbf{V}^{0}$ corresponds to the set of price indices associated with the maximum objective function value. Because $\mathbf{V}^{0}$ is an extreme point of the expansion of the previous simplex, the new simplex has the same orientation as the previous simplex.

$$
\begin{aligned}
& \text { for } j=1 \text { to } n \text { do } \\
& \qquad \mathbf{V}^{j}=\left\{\begin{array}{ll}
\mathbf{V}^{0}+\mathbf{e}_{j} & \text { with probability } 0.5 \\
\mathbf{V}^{0}-\mathbf{e}_{j} & \text { with probability } 0.5
\end{array}\right. \text { // Establish the new simplex. } \\
& \text { end for }
\end{aligned}
$$

When establishing a new simplex, the boundary conditions of this simplex should be examined, as described in Step 3. With a new simplex in hand, the heuristic returns to Step 3.

\section{Numerical Analysis}

To demonstrate the validity of our model, as well as the effectiveness of the proposed solution approach, a numerical analysis has been conducted. The numerical analysis is designed to capture the randomness in the demand and the cross-price sensitivity relationship in our examples.

The stochastic nature of demand may be incorporated in either an additive or multiplicative sense. As such, we consider the following demand functions in our numerical experiments:

$$
D_{i}(\mathbf{p})=d_{i}(\mathbf{p})+\epsilon_{i} \text { and } D_{i}(\mathbf{p})=d_{i}(\mathbf{p}) \epsilon_{i}
$$

where $d_{i}(\mathbf{p})=\alpha_{i}+\sum_{j=1}^{n} \beta_{i j} p_{j}$ represents the deterministic component of demand and $\epsilon_{i}$ represents the stochastic component of demand. Here, $\alpha_{i}$ represents the market potential of product $i\left(\alpha_{i}>0\right)$, and $\beta$ is a matrix of price sensitivity coefficients such that $\beta_{i i}$ represents the own-price sensitivity of product $i$ and $\beta_{i j}$ represents the cross-price sensitivity between products $i$ and $j$. Because demand for product $i$ is expected to decrease if its selling price increases, $\beta_{i i}<0$. If products $i$ and $j$ are substitutes, $\beta_{i j}>0$ to indicate that demand for product $i$ will increase if the price of substitute product $j$ increases. Similarly, if $i$ and $j$ are complementary products, $\beta_{i j}<0$ to reflect decreased demand for product $i$ if the price of product $j$ increases. The expected 


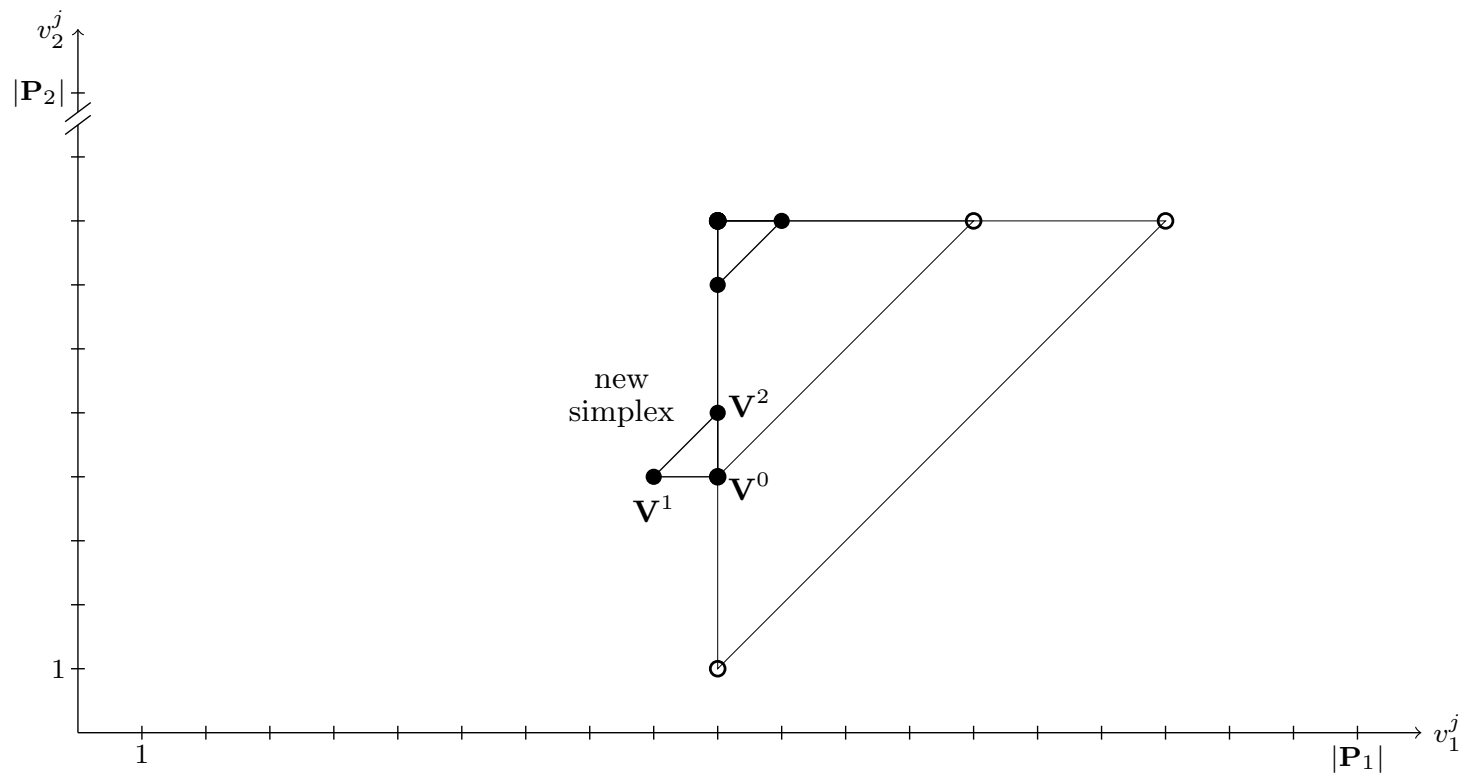

Figure 5: Vertex $\mathbf{V}^{0}$ corresponds to the set of price indices associated with the maximum objective function value. Because $\mathbf{V}^{0}$ is not an extreme point of the expansion of the previous simplex, the new simplex is randomly rotated about the new anchor $\mathbf{V}^{0}$.

demand for product $i, \mathrm{E}\left[D_{i}(\mathbf{p})\right]$, is defined to equal $d_{i}(\mathbf{p})$. This form of the demand function is common in the pricing literature (c.f., Talluri and Van Ryzin (2005)). We choose this form for its ease of explanation and its widespread use.

In what follows, we investigate four particular demand functions involving linear demand - normallydistributed additive uncertainty, normally-distributed multiplicative uncertainty, uniformly-distributed additive uncertainty, and uniformly-distributed multiplicative uncertainty. Although we conducted the numerical analysis for these particular demand forms, our model and solution approach are applicable to other demand functions with the following (reasonable) restrictions. First, the demand function must be "well-behaved" (demand decreases as own-prices increase, demand increases as other prices increase, and overall demand must not increase if the price of any product increases). Second, the stochastic component of demand, $\epsilon_{i}$, must be bounded. Third the expected demand should equal the deterministic component of the demand function (i.e., $\mathrm{E}\left[\epsilon_{i}\right]=0$ for additive uncertainty and $\mathrm{E}\left[\epsilon_{i}\right]=1$ for multiplicative uncertainty).

Linear demand with normal additive uncertainty. In the case of linear demand with normally-distributed additive uncertainty, we assume that $\epsilon_{i} \sim \mathcal{N}\left(0, \sigma_{i}\right)$, with the added restriction that $\epsilon_{i}$ is bounded. As such 
$\epsilon_{i} \in[-3 \sigma, 3 \sigma]$. The corresponding CDF of demand, $F_{i}(\mathbf{p}, d)$, is given by

$$
\begin{aligned}
F_{i}(\mathbf{p}, d) & \equiv \operatorname{Pr}\left\{D_{i}(\mathbf{p}) \leq d\right\} \\
& =\operatorname{Pr}\left\{\alpha_{i}+\sum_{j=1}^{n} \beta_{i j} p_{j}+\epsilon_{i} \leq d\right\} \\
& =\operatorname{Pr}\left\{\epsilon_{i} \leq d-\alpha_{i}-\sum_{j=1}^{n} \beta_{i j} p_{j}\right\} \\
& =\operatorname{Pr}\left\{\frac{\epsilon_{i}}{\sigma} \leq \frac{d-\alpha_{i}-\sum_{j=1}^{n} \beta_{i j} p_{j}}{\sigma}\right\} \\
& = \begin{cases}0 & \text { if } d<\mathrm{E}\left[D_{i}(\mathbf{p})\right]-3 \sigma_{i} \\
& \text { if } \mathrm{E}\left[D_{i}(\mathbf{p})\right]-3 \sigma_{i} \leq d \leq \mathrm{E}\left[D_{i}(\mathbf{p})\right]+3 \sigma_{i} \\
1 & \text { if } d>\mathrm{E}\left[D_{i}(\mathbf{p})\right]+3 \sigma_{i}\end{cases}
\end{aligned}
$$

Linear demand with normal multiplicative uncertainty. For normally-distributed multiplicative uncertainty, where $\epsilon_{i} \sim \mathcal{N}\left(1, \sigma_{i}\right)$, and the deterministic component of demand is linear, $F_{i}(\mathbf{p}, d)$ is given by

$$
F_{i}(\mathbf{p}, d)= \begin{cases}0 & d<\mathrm{E}\left[D_{i}(\mathbf{p})\right]\left(1-3 \sigma_{i}\right) \\ \Phi\left(\frac{\frac{d}{\alpha_{i}-\sum_{j=1}^{n} \beta_{i j} p_{j}}-1}{\sigma}\right) & \mathrm{E}\left[D_{i}(\mathbf{p})\right]\left(1-3 \sigma_{i}\right) \leq d \leq \mathrm{E}\left[D_{i}(\mathbf{p})\right]\left(1+3 \sigma_{i}\right) \\ 1 & d>\mathrm{E}\left[D_{i}(\mathbf{p})\right]\left(1+3 \sigma_{i}\right)\end{cases}
$$

As in the case of normally-distributed demand with additive uncertainty, we consider $\epsilon_{i}$ to be restricted within $\pm 3 \sigma_{i}$.

Linear demand with uniform additive uncertainty. For uniformly-distributed additive uncertainty, we consider $\epsilon_{i} \sim \operatorname{unif}\left(a_{i}, b_{i}\right)$, where $E\left[\epsilon_{i}\right]=0$ (i.e., $a_{i}=-b_{i}$ ). The resulting CDF of demand for product $i$, given linear expected demand, is as follows:

$$
F_{i}(\mathbf{p}, d)= \begin{cases}0 & d<\mathrm{E}\left[D_{i}(\mathbf{p})\right]+a_{i} \\ \frac{d-\left(\alpha_{i}+\sum_{j=1}^{n} \beta_{i j} p_{j}\right)-a_{i}}{b_{i}-a_{i}} & \mathrm{E}\left[D_{i}(\mathbf{p})\right]+a_{i} \leq d \leq \mathrm{E}\left[D_{i}(\mathbf{p})\right]+b_{i} \\ 1 & d>\mathrm{E}\left[D_{i}(\mathbf{p})\right]+b_{i}\end{cases}
$$

Linear demand with uniform multiplicative uncertainty. Finally, for linear demand with uniformly-distributed multiplicative uncertainty, where $\epsilon_{i} \sim \operatorname{unif}\left(a_{i}, b_{i}\right)$ and $E\left[\epsilon_{i}\right]=1$, we have

$$
F_{i}(\mathbf{p}, d)= \begin{cases}0 & d<\mathrm{E}\left[D_{i}(\mathbf{p})\right] a_{i} \\ \frac{d}{\alpha_{i}+\sum_{j=1}^{n} \beta_{i j} p_{j}}-a_{i} & \mathrm{E}\left[D_{i}(\mathbf{p})\right] a_{i} \leq d \leq \mathrm{E}\left[D_{i}(\mathbf{p})\right] b_{i} \\ b_{i}-a_{i} & d>\mathrm{E}\left[D_{i}(\mathbf{p})\right] b_{i}\end{cases}
$$




\subsection{Generation of Sample Problems}

In the absence of suitable benchmark problems in the literature, we generated random problem instances to evaluate the effectiveness and efficiency of the proposed solution approach. In these problems, $c_{i}$, the procurement cost of product $i$, was uniformly distributed in [1,5] and the holding cost, $h_{i}$, was in the range of 5-15\% of $c_{i} . \mathbf{P}_{i}$, the set of allowable selling prices for product $i$, was constructed such that the minimum value in the set $\mathbf{P}_{i}$ is in the range $\left[1.25 c_{i}, 3 c_{i}\right]$. These prices increase in increments between $\$ 0.01$ and $\$ 0.10$ until a pre-specified number of candidate prices are reached (as described in the numerical analysis below).

All of the four aforementioned forms of linear demand were considered in these problems. As such, cross-price sensitivities, $\beta_{i j}$, are in the range $[0.1,2.0]$ and own-price sensitivities, $\beta_{i i}$, are in the range $\left[-1 \sum_{\substack{j=1 \\ j \neq i}}^{n} \beta_{i j},-2 \sum_{\substack{j=1 \\ j \neq i}}^{n} \beta_{i j}\right]$. Thus, as the price of product $j$ increases, the demand for product $i$ also increases. However, if the price of product $i$ increases, the demand for that product decreases. The market potential, $\alpha_{i}$, for product $i$, was chosen to be between 1.5 and 4 times the minimum value of the price-sensitive portion of demand, which occurs when products $j$ are sold at their lowest prices and product $i$ is sold at its highest price.

$L_{i}$, the lower bound on the procurement quantity for product $i$, was selected to be between $25-75 \%$ of the expected demand when all products are offered at their mean allowable price. Similarly, the upper bound, $U_{i}$, was selected to be in $75-200 \%$ of expected demand.

Two additional resource constraints were considered in these problems, such that $R_{1}$ represents a budget limitation and $R_{2}$ represents a space (volume) limitation. To ensure the feasibility of each test problem, the available budget was determined to be large enough to allow the retailer to purchase at least the minimum required quantity of each product. To ensure that the budget constraint would be binding, the available budget was limited by the maximum allowable quantity of each product. Thus, the total budget, $R_{1}$ was chosen from the range $\left[\sum_{i=1}^{n} L_{i} c_{i}, \sum_{i=1}^{n} U_{i} c_{i}\right]$. The volume of each product was chosen such that $r_{i, 2}$ was in the range [1,5], where $r_{i, 2}$ is the amount of resource 2 (volume) consumed by product $i$. Similar to the determination of the budget value, the total available volume was calculated such that $R_{2}$ is in the interval $\left[\sum_{i=1}^{n} L_{i} r_{i, 2}, \sum_{i=1}^{n} U_{i} r_{i, 2}\right]$.

Parameters describing the uncertainty of demand were chosen according to the form of the demand function. In the case of uniform additive uncertainty, where $\epsilon_{i} \sim \operatorname{unif}\left(a_{i}, b_{i}\right), b_{i}$ was chosen to be between $10 \%$ and $30 \%$ of the expected demand when all products are offered at their mean allowable prices, and $a_{i}=-b_{i}$. The value of $b_{i}$ was similarly determined for the case of uniform multiplicative uncertainty, although $a_{i}=2-b_{i}$ to ensure that $E\left[\epsilon_{i}\right]=1$. For the case of normally-distributed uncertainty, where $\epsilon_{i} \sim \mathcal{N}\left(\mu_{i}, \sigma_{i}\right), \mu_{i}=0\left(\mu_{i}=1\right)$ and $\sigma_{i}=b_{i} / 3$ for the additive (multiplicative) form of uncertainty.

In the remainder of this section, we describe a numerical analysis of problems of "small" and "large" scale. The small-scale problems were chosen to be of sufficiently diminutive scale to allow optimal solutions to be obtained via exhaustive enumeration, thus affording a means for evaluating the performance of our heuristic against optimality. The size of the large-scale problems reflect realistic numbers of stock keeping units (SKUs) that may be found within a typical product category at retail supermarkets. For example, Russell and Petersen (2000) indicate this number to be about 50. Our direct information from a collaborating large regional supermarket chain in the Northeast USA also suggests that the number of SKUs in a category rarely exceeds 100 . 


\subsection{Computational Experience: Small-scale Problems}

To assess the effectiveness of our proposed heuristic, we tested our approach on three sets of problems. These problems involved 3 products with 20 prices per product, 5 products with 7 prices per product, and 10 products with 3 prices per product. Due to the small size of our test problems, it was possible to exhaustively enumerate all price combinations, solving problem MIP-2 for each unique combination and ultimately determining the optimal solution.

For each set of problems we investigated the four forms of demand previously described in this section (additive normally distributed uncertainty, multiplicative normal, additive uniform, and multiplicative uniform). Forty random problem instances were generated for each of the three problem sets, giving us a total of 120 test problems. Each test problem was restarted $n$ times (e.g., Step 1 of the heuristic was applied three times for the test problems involving three products).

Table 1 contains a performance summary of our heuristic on these small-scale test problems. The third column shows the total number of allowable price combinations that are considered via exhaustive enumeration. In general, the total number of distinct price combinations is $\prod_{i=1}^{n}\left|\mathbf{P}_{i}\right|$. The fourth column contains the average number of price combinations that were investigated by the proposed solution approach. The "\% of Soln Space" column is the fraction of the average number of solutions investigated by the heuristic over the total number of price combinations. The average gap between the heuristic and optimal objective function values is less than $1.1 \%$ across all three sets of problems, indicating that the proposed heuristic is able to find high-quality solutions by exploring only a small percentage of the solution space. As shown in the final column, the average runtime of the heuristic are quite reasonable.

Table 1: Summary of performance metrics for small problems.

\begin{tabular}{ccccccc}
\hline Set & $\begin{array}{c}\text { Problem } \\
\text { Size }\end{array}$ & $\begin{array}{c}\text { Price } \\
\text { Combinations }\end{array}$ & $\begin{array}{c}\text { Avg \# of Solns } \\
\text { Investigated }\end{array}$ & $\begin{array}{c}\text { \% of Soln } \\
\text { Space }\end{array}$ & $\begin{array}{c}\text { Avg } \\
\text { Gap }\end{array}$ & $\begin{array}{c}\text { Avg Total } \\
\text { Soln Time [s] }\end{array}$ \\
\hline 1 & $n=3,\left|P_{i}\right|=20$ & 8,000 & 179.7 & $2.2 \%$ & $1.1 \%$ & 0.94 \\
2 & $n=5,\left|P_{i}\right|=7$ & 16,807 & 605.9 & $3.6 \%$ & $0.2 \%$ & 5.23 \\
3 & $n=10,\left|P_{i}\right|=3$ & 59,049 & 2420.8 & $4.1 \%$ & $0.1 \%$ & 40.11 \\
\hline
\end{tabular}

In Table 2, we describe the heuristic's performance for test problems in which the heuristic did not find an optimal solution. Although the percentage of problems for which the heuristic found the global optimal solution appears to be low $(\leq 70 \%)$, the average gap between the heuristic and optimal objective function values is less than $1.1 \%$ across all three sets of problems. Additionally, if we focus solely on those problems for which the heuristic did not find the optimal solution, the average gap rose to a value that is still less than $3 \%$ from optimal.

Table 2: Analysis of sub-optimal solutions for small problems.

\begin{tabular}{cccc}
\hline $\begin{array}{c}\text { Problem } \\
\text { Size }\end{array}$ & $\begin{array}{c}\text { \% of Solns } \\
\text { Optimal }\end{array}$ & $\begin{array}{c}\text { Max } \\
\text { Gap }\end{array}$ & $\begin{array}{c}\text { Avg Gap } \\
\text { for Sub-opt }\end{array}$ \\
\hline$n=3,\left|P_{i}\right|=20$ & $60.0 \%$ & $10.6 \%$ & $2.6 \%$ \\
$n=5,\left|P_{i}\right|=7$ & $70.0 \%$ & $2.1 \%$ & $0.7 \%$ \\
$n=10,\left|P_{i}\right|=3$ & $62.5 \%$ & $0.8 \%$ & $0.1 \%$ \\
\hline
\end{tabular}

All computational experiments were conducted on a desktop PC with eight Intel Core i7 2.8Ghz processors running Ubuntu Linux 10.10. The heuristic was programmed in C, and the integer program MIP-2 was solved using CPLEX 12. 


\subsection{Computational Experience: Large-scale Problems}

For large-scale problems, exhaustive enumeration of all possible price combinations is impractical. In lieu of alternative methods for determining optimal solutions to problems of this scale, we provide, in Table 3 , a summary of the heuristic's computational efficiency for test problems involving up to 100 products. These test problems were generated using the same procedure as the small-scale problems. For each of the 3 sets of problems, 12 distinct problem instances were created, resulting in 36 unique problems. Due to the enormity of the solution space, as highlighted in the third column of the table ("Price Combinations"), attempts to explicitly investigate a significant percentage of the solution space would be time-prohibitive. Instead, we limited the runtimes for each problem to 30-minutes. In this table, a "solution" describes a candidate price vector as well as the corresponding optimal allocation quantities (as determined by the solution to problem MIP-2). The "average time per solution" metric, which captures the time required to solve MIP-2 for each candidate price vector, indicates that even though the solution space is extremely large, each candidate solution can be evaluated quite quickly. Thus, although the runtimes-per-solution increase with problem size, the underlying integer program that is used to solve problem MIP-2 is still solvable in a reasonable amount of time.

Table 3: Performance metrics for large problems.

\begin{tabular}{ccccc}
\hline Set & $\begin{array}{c}\text { Problem } \\
\text { Size }\end{array}$ & $\begin{array}{c}\text { Price } \\
\text { Combinations }\end{array}$ & $\begin{array}{c}\text { Avg \# of Solns } \\
\text { Investigated }\end{array}$ & $\begin{array}{c}\text { Avg Time } \\
\text { per Soln }[\mathbf{s}]\end{array}$ \\
\hline 1 & $n=25,\left|P_{i}\right|=5$ & $\sim 2.98 \times 10^{17}$ & $26,400.3$ & 0.07 \\
2 & $n=50,\left|P_{i}\right|=5$ & $\sim 8.88 \times 10^{34}$ & $6,230.8$ & 0.32 \\
3 & $n=100,\left|P_{i}\right|=5$ & $\sim 7.89 \times 10^{69}$ & $1,301.0$ & 2.00 \\
\hline
\end{tabular}

\section{Conclusions and Opportunities for Future Research}

While the newsvendor (or newsboy) is a long-standing problem in operations management (Edgeworth, 1888), its application to price-setting has remained largely restricted to single product problems. Numerous extensions of the newsvendor that consider a variety of other modeling assumptions have been studied in the literature because, like the EOQ (economic ordering quantity) formula, it continues to be the workhorse of many a supply chain management software and planning activity. However, to the best of our knowledge, this is the first work to address a newsvendor interested in: (i) selling multiple products whose demands are stochastic and display a cross-elasticity relationship, and (ii) determining the prices along with the order quantities. Furthermore, our work also seeks to generate integer-valued solutions that can be implemented by real-world retailers and incorporated within modern supply chain management software packages.

Our work is closest in spirit to that of Shi et al. (2011), as both represent novel developments in this research field by seeking to solve a price-setting newsvendor that considers multiple products. However, our work was primarily motivated by a need to account for cross-price elasticity of demand, which captures the economic realities of budget-sensitive customers in the current economy, as well as by the issue of generating integer-valued implementable solutions. Incorporation of cross-price elasticity into our demand structure leads to a computational challenge, since the resulting objective function is not conducive to optimization via classical methods of integer or non-linear programming. Our numeric technique is able to surmount this challenge and produce encouraging results on small-scale and large-scale problems. We believe our research not only paves the way for generating effective solutions for this difficult problem, but also opens the avenue for further research along the lines of stochastic demand, multiple products, and cross-price elasticity of demand.

One advantage of our solution approach lies in its flexibility to solve problems with a variety of demand functions. However, future research should focus on more problem-specific heuristics that can exploit the 
structural properties of particular problems. Another interesting line of future research would be to focus on the optimal size of the product assortment being offered by a retailer. As noted earlier in our model development, here we make the typical assumption made in the literature on optimizing product procurement quantities that the size $n$ of the product assortment offered by the retailer in the focal category has already been selected. So, this paper like the existing literature (e.g., Cachon et al. (2005); Aydin and Porteus (2008); Murray et al. (2010); Martínez-de-Albéniz and Roels (2011)) has treated optimizing product assortment size and product procurement quantities as related but sequential decision problems by a retailer. The challenge for future research would be to integrate them as a simultaneous decision problem on the part of the retailer.

\section{Acknowledgements}

The authors would like to acknowledge George Ruff for his work on coding the proposed solution method. They are also grateful to the two anonymous reviewers for their very constructive feedback and suggestions on the earlier versions of this manuscript.

\section{References}

Abdel-Malek, L., Areeratchakul, N.. A quadratic programming approach to the multi-product newsvendor problem with side constraints. European Journal of Operational Research 2007;176(3):1607 - 1619.

Abdel-Malek, L., Montanari, R.. An analysis of the multi-product newsboy problem with a budget constraint. International Journal of Production Economics 2005a;97(3):296 - 307.

Abdel-Malek, L., Montanari, R.. On the multi-product newsboy problem with two constraints. Computers and Operations Research 2005b;32(8):2095-2116.

Abdel-Malek, L., Montanari, R., Meneghetti, D.. The capacitated newsboy problem with random yield: The gardener problem. International Journal of Production Economics 2008;115(1):113 - 127.

Abdel-Malek, L., Montanari, R., Morales, L.. Exact, approximate, and generic iterative models for the multi-product newsboy problem with budget constraint. International Journal of Production Economics 2004;91(2):189 - 198.

Agrawal, V., Seshadri, S.. Impact of uncertainty and risk aversion on price and order quantity in the newsvendor problem. Manufacturing \& Service Operations Management 2000;2(4):410-423.

Ailawadi, K.L., Zhang, J., Krishna, A., Kruger, M.W.. When wal-mart enters: How incumbent retailers react and how this affects their sales outcomes. Journal of Marketing Research 2010;47(4):577-593.

Aydin, G., Porteus, E.. Joint inventory and pricing decisions for an assortment. Operations Research 2008;56(5):1247-1255.

Ball, D.. Consumer-goods firms duel for shelf space. Wall Street Journal 2004;

Bijmolt, T., Heerde, H.V., Pieters, R.. New empirical generalizations on the determinants of price elasticity. Journal of Marketing Research 2005;38:141-156.

Bisi, A., Dada, M.. Dynamic learning, pricing, and ordering by a censored newsvendor. Naval Research Logistics 2007;54(4):448-461.

Cachon, G., Kök, A.. Implementation of the newsvendor model with clearance pricing: How to (and how not to) estimate a salvage value. Manufacturing \& Service Operations Management 2007;9(3):176-290. 
Cachon, G., Terwiesch, C., Xu, Y.. Retail assortment planning in the presence of consumer search. Manufacturing \& Service Operations Management 2005;7(4):330 - 346.

Chen, J., Bell, P.. The impact of customer returns on pricing and order decisions. European Journal of Operational Research 2009;195(1):280-295.

Chen, Y., Xu, M., Zhang, Z.. Technical Note-A Risk-Averse Newsvendor Model Under the CVaR Criterion. Operations Research 2009;57(4):1040-1044.

Clifford, S.. Stores scramble to accommodate budget shoppers. New York Times 2010;Sept 21.

Corman, F., D’Ariano, A., Pacciarelli, D., Pranzo, M.. A tabu search algorithm for rerouting trains during rail operations. Transportation Research Part B: Methodological 2010;44(1):175-192.

Deloitte, . A walk down the grocery aisle: Executive survey results exploring private label and national brands. 2010.

Duvvuri, S., Ansari, A., Gupta, S.. Consumers' price sensitivities across complementary categories. Management Science 2007;53(12):1933-1945.

Edgeworth, F.. The mathematical theory of banking. JR Stat Soc 1888;53:113-127.

Flatters, P., Willmott, M.. Understanding the post-recession consumer. Harvard Business Review $2009 ;: 106-112$.

Fry, M., Kapuscinski, R., Olsen, T.. Coordinating production and delivery under a (z, Z)-type vendormanaged inventory contract. Manufacturing \& Service Operations Management 2001;3(2):151-173.

Gauri, D., Sudhir, K., Talukdar, D.. The temporal and spatial dimensions of price search: Insights from matching household survey and purchase data. Journal of Marketing Research 2008;45:226-240.

Geyskens, I., Gielens, K., Gijsbrechts, E.. Proliferating private-label portfolios: How introducing economy and premium private labels influences brand choice. Journal of Marketing Research 2010;47(5):791-807.

Greenhouse, S.. How costco became the anti-wal-mart. 2005.

Hadley, G., Whitin, T.. Analysis of Inventory Systems. Prentice Hall, 1963.

van Heerde, H., Leeflang, P., Wittink, D.. Decomposing the sales promotion bump with store data. Marketing Science 2004;23(3):317-334.

Khouja, M.. The single-period (news-vendor) problem: literature review and suggestions for future research. Omega 1999;27(5):537 - 553.

Lau, H., Lau, A.. The multi-product multi-constraint newsboy problem: Applications, formulation and solution. Journal of Operations Management 1995;13(2):153 - 162.

Lau, H., Lau, A.. Some results on implementing a multi-item multi-constraint single-period inventory model. International Journal of Production Economics 1997;48(2):121 - 128.

Levy, M., Grewal, D., Kopalle, P., Hess, J.. Emerging trends in retail pricing practice: implications for research. Journal of Retailing 2004;80(3):xiii-xxi.

Leung, C.W., Wong, T.N., Mak, K.L., Fung, R.Y.K.. Integrated process planning and scheduling by an agent-based ant colony optimization. Computers \& Industrial Engineering 2010;59(1):166-180. 
Martínez-de-Albéniz, V., Roels, G.. Competing for shelf space. Production and Operations Management 2011;20(1):32-46.

McAlister, L., George, E., Chien, Y.. A basket-mix model to identify cherry-picked brands. Journal of Retailing 2009;85(4):425-436.

Moin, N.H., Salhi, S., Aziz, N.A.B.. An efficient hybrid genetic algorithm for the multi-product multiperiod inventory routing problem. International Journal of Production Economics 2011;133(1):334-343.

Monahan, G., Petruzzi, N., Zhao, W.. The Dynamic Pricing Problem from a Newsvendor's Perspective. Manufacturing \& Service Operations Management 2004;6(1):73-91.

Moon, I., Silver, E.A.. The multi-item newsvendor problem with a budget constraint and fixed ordering costs. The Journal of the Operational Research Society 2000;51(5):602 - 608.

Murray, C., Talukdar, D., Gosavi, A.. Joint optimization of product price, display orientation and shelfspace allocation in retail category management. Journal of Retailing 2010;86(2):125-136.

Nelder, J., Mead, R.. A simplex method for function minimization. The Computer Journal 1965;7(4):308313.

Niederhoff, J.. Using separable programming to solve the multi-product multiple ex-ante constraint newsvendor problem and extensions. European Journal of Operational Research 2007;176(2):941 - 955.

Nielsen, . Promotions drive 35\% of storewide sales, more at the holidays. 2008.

Özler, A., Tan, B., Karaesmen, F.. Multi-product newsvendor problem with value-at-risk considerations. International Journal of Production Economics 2009;117(2):244 - 255.

Petruzzi, N., Dada, M.. Pricing and the newsvendor problem: A review with extensions. Operations Research 1999;47(2):183-194.

Russell, G., Petersen, A.. Analysis of cross category dependence in market basket selection. Journal of Retailing 2000;76(3):367-392.

Shi, C., Zhao, X., Xia, Y.. The setting of profit targets for target oriented divisions. European Journal of Operational Research 2010;206(1):86 - 92.

Shi, J., Zhang, G., Sha, J.. Jointly pricing and ordering for a multi-product multi-constraint newsvendor problem with supplier quantity discounts. Applied Mathematical Modelling 2011;35(6):3001 - 3011.

Taleizadeh, A., Niaki, S., Hoseini, V.. Optimizing the multi-product, multi-constraint, bi-objective newsboy problem with discount by a hybrid method of goal programming and genetic algorithm. Engineering Optimization 2009;41(5):437-457.

Talluri, K., Van Ryzin, G.. The theory and practice of revenue management. Springer Verlag, 2005.

Talukdar, D., Gauri, D., Grewal, D.. An empirical analysis of the extreme cherry picking behavior of consumers in the frequently purchased goods market. Journal of Retailing 2010;86(4):336-354.

Tarnowski, J.. Growing, naturally. Progressive Grocer 2007;86(13):70-71.

Torczon, V.. On the convergence of the multidirectional search algorithm. SIAM Journal on Optimization 1991;1(1):123 - 145 . 
Whitin, T.. Inventory control and price theory. Management Science 1955;2(1):61-68.

Wong, W., Qi, J., Leung, S.. Coordinating supply chains with sales rebate contracts and vendor-managed inventory. International Journal of Production Economics 2009;120(1):151-161.

$\mathrm{Xu}, \mathrm{M}$. . A price-setting newsvendor model under cvar decision criterion with emergency procurement. Journal of Systems Science and Systems Engineering 2010;19(1):85-104.

Yang, M., Chen, W.. A study on shelf space allocation and management. International journal of production economics 1999;60:309-317.

Yang, S., Shi, C., Zhao, X.. Optimal ordering and pricing decisions for a target oriented newsvendor. Omega 2010;In Press, Corrected Proof:-.

Yano, C., Gilbert, S.. Coordinated pricing and production/procurement decisions: A review. In: Eliashberg, J., Chakravarty, A., editors. Managing Business Interfaces. Springer US; volume 16 of International Series in Quantitative Marketing; 2005. p. 65-103.

Zhang, B., Xu, X., Hua, Z.. A binary solution method for the multi-product newsboy problem with budget constraint. International Journal of Production Economics 2009;117(1):136 - 141.

Zhang, D., Liu, R., M'Hallah, R., Leung, S.C.H.. A simulated annealing with a new neighborhood structure based algorithm for high school timetabling problems. European Journal of Operational Research 2010;203(3):550-558.

Zhang, G.. The multi-product newsboy problem with supplier quantity discounts and a budget constraint. European Journal of Operational Research 2010;206(2):350 - 360.

Zhu, T., Singh, V., Manuszak, M.. Market structure and competition in the retail discount industry. Journal of Marketing Research 2009;46(4):453-466. 